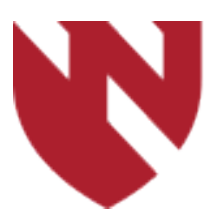

December 2019

\title{
Identifying Current Opioid Prescription Practice and Implementing Guidelines after Total Knee and Total Hip Arthroplasty
}

\author{
Gordon Roedel \\ University of Nebraska Medical Center \\ Phillip Thomas \\ University of Nebraska Medical Center \\ Curtis Hartman \\ University of Nebraska Medical Center \\ Kevin Garvin \\ University of Nebraska Medical Center \\ Beau Konigsberg \\ University of Nebraska Medical Center
}

Tell us how you used this information in this short survey.

Follow this and additional works at: https://digitalcommons.unmc.edu/gmerj

Part of the Higher Education Commons, and the Medicine and Health Sciences Commons

\section{Recommended Citation}

Roedel, G., Thomas, P., Hartman, C., Garvin, K., , Konigsberg, B. Identifying Current Opioid Prescription Practice and Implementing Guidelines after Total Knee and Total Hip Arthroplasty. Graduate Medical Education Research Journal. 2019 Dec 13; 1(1).

https://digitalcommons.unmc.edu/gmerj/vol1/iss1/38

This Conference Proceeding is brought to you for free and open access by DigitalCommons@UNMC. It has been accepted for inclusion in Graduate Medical Education Research Journal by an authorized editor of DigitalCommons@UNMC.For more information, please contact digitalcommons@unmc.edu. 
Identifying Current Opioid Prescription Practice and Implementing Guidelines after Total Knee and Total Hip Arthroplasty

\section{Creative Commons License}

\section{c) (1)@ $\Theta$}

This work is licensed under a Creative Commons Attribution-Noncommercial-No Derivative Works 4.0 License. 


\section{Does Prophylactic Administration of TXA Reduce Mean Operative Time and Postoperative Blood Loss in Posterior Approach Lumbar Spinal Fusion Surgery Performed for Degenerative Spinal Disease?}

Evan Larson, Emmett Gannon, Tyler Evans, Jake Long, Elizabeth Lyden, Chris Cornett

Mentor: Chris Cornett

Program: Orthopaedic Surgery

Objective: To investigate association between prophylactic TXA administration prior to one and two-level posterior lumbar interbody fusion operations and perioperative blood loss, mean operative time, postoperative transfusion, and postoperative venous thromboembolic events.

Summary of Background Data: Tranexamic acid (TXA) is a systemic anti-fibrinolytic that competitively inhibits lysine binding sites on plasminogen, reversibly blocking its binding to fibrin and impeding fibrinolysis and clot degradation. TXA's role in routine spinal surgery remains poorly described. Most spinal literature on perioperative TXA administration has considered operations performed for major adult and pediatric spinal deformity.

Methods: Two groups, a study group composed of 75 patients who underwent one and two-level posterior lumbar interbody fusion operations for degenerative indications who received TXA prior to the start of the procedure, and a control group composed of 75 patients who underwent similar surgeries for the same indications and did not receive TXA preoperatively, were retrospectively enrolled. Demographic, laboratory, and surgical data were collected and analyzed.
Results: Statistically significant reductions were noted in the TXA group in postoperative day 1 drain output ( $\mathrm{p}<0.0041)$, total postoperative drain output $(\mathrm{p}=0.027)$, and mean surgical time $(\mathrm{p}<0.0001)$.

Conclusions: Based on the results of the current study, we feel TXA's routine use should be considered in one and two- level posterior lumbar fusion operations performed for degenerative indications, as it is safe to administer routinely and associated with reduced postoperative drain output and mean surgical time.

https://doi.org/10.32873/unmc.dc.gmerj.1.1.036

\section{Identifying Current Opioid Prescription Practice and Implementing Guidelines after Hand and Upper Extremity Surgery - A Project in Quality Improvement}

Ryan E Miller, David Brown, Mark Amirtharaj, Brandon Bennett, Todd Gilbert, CJ Hansen, Evan Larson, Kent Rinehart, Daniel Firestone, Joseph Morgan, Philipp Streubel, Matthew Teusink

\section{Mentor: Daniel Firestone}

Program: Orthopaedic Surgery

Background \& Objectives: Opioid pain medications prescriptions following upper extremity surgery is part of the standard of care. However, there is a growing concern about opioid abuse, overdose deaths, and diversion of opioids in the community. New evidence suggesting opioid overprescription has prompted a closer look at current practice. Objectives of this quality improvement project are 1) to define prescribing habits and patient utilization of opioids following upper extremity surgery within our institution, 2) to develop prescription guidelines, and 3 ) to educate patients about opioid usage and disposal.
Methods: Stage I consists of collecting prospective data from patients undergoing upper extremity surgery by 4 upper extremity surgeons over 6 consecutive months. Data are gathered from the electronic medical record and survey at clinic visits up to 90 days postoperatively including level of pain, opioid pills prescribed (converted to morphine equivalents), number of unused pills, and date of last opioid use. Patients are asked whether they were educated on pill disposal. Results will be used to develop prescription guidelines and patient education protocols

Stage II consists of collecting prospective data from patients undergoing upper extremity surgery by 4 upper extremity surgeons over 6 consecutive months. Postoperative opioid prescriptions guided by previous results will be provided with standardized education on opioid disposal. The aforementioned data will again be collected at clinic visits up to 90 days postoperatively. Data from each stage will then be compared to assess the effectiveness of the implemented guidelines.

Results \& Conclusions: Pending further data collection.

https://doi.org/10.32873/unmc.dc.gmerj.1.1.037

\section{Identifying Current Opioid Prescription Practice and Implementing Guidelines after Total Knee and Total Hip Arthroplasty \\ Gordon Roedel, Phillip Thomas, Curtis Hartman, Kevin Garvin, Beau Konigsberg}

Mentor: Curtis Hartman

Program: Orthopaedic Surgery

Background \& Objectives: Opioid pain medications prescribed following total hip arthroplasty (THA) and total knee arthroplasty (TKA) are critical to postoperative pain management. However, a lack of evidence regarding appropriate prescription practices can contribute to overprescribing and diversion of opioids within the community. Objectives of this quality improvement project are 1) to understand prescribing habits and patient utilization of opioids following THA and TKA within our institution, 2) develop appropriate prescription guidelines, and 3) improve patient knowledge regarding unused opioid disposal.

Methods: Stage I consists of collecting prospective data from opioid-naïve patients undergoing primary THA and TKA over 6 consecutive months. Data are gathered from the electronic medical record and survey at clinic visits up to 90 days postoperatively including level of pain, number/type of opioid pills prescribed postoperatively (converted to morphine equivalents), number of unused pills, and date of last opioid use. Patients are asked whether they were educated on disposal of unused opioids. Results will be used to develop opioid prescription guidelines and patient education protocols which will be implemented during Stage II.

Stage II consists of collecting prospective data from opioid-naïve patients undergoing primary THA and TKA over 6 consecutive months. Postoperative opioid prescriptions guided by previous results will be provided with standardized education on opioid disposal. The aforementioned data will again 
be collected at clinic visits up to 90 days postoperatively. Data from each stage will be compared to assess the effectiveness of the guidelines in providing adequate analgesia while minimizing opioid prescriptions.

Results \& Conclusions: Pending further data collection.

https://doi.org/10.32873/unmc.dc.gmerj.1.1.038

\section{An Immediate Weight Bearing Protocol versus Stress Radiography to Determine Ankle Stability in Patients with Isolated SER Distal Fibula Fractures \\ Phillip Thomas, Leonid Grossman, Justin Siebler, Ivan Tarkin, Lori Reed, Liz Lyden, Matthew Mormino}

Mentor: Matthew Mormino

Program: Orthopaedic Surgery

Purpose: To compare stress radiographs with weight bearing radiographs in the acutely injured ankle and determine if stress views may be unnecessary and ultimately lead to unnecessary surgical intervention.

To determine if patients allowed to weight bear will show similar results radiographically and clinically to the current gold standard stress views.

Methods: A prospective study will be conducted with UNMC, UPMC \& Mississippi
Centers with 200 total subjects enrolled. Every subject with isolated fibular fracture will have stress view $\mathrm{x}$-rays taken in the ED. They will then be placed in a CAM boot and follow up in clinic $\sim 7$ days later. Weight bearing X-rays will be obtained at follow up and UNMC \& UPMC will use weight bearing $\mathrm{X}$-rays to determine operative intervention. Mississippi will use only stress views to determine operative intervention

Results \& Conclusions: Collecting data. Currently nine subjects had medial clear space widening $>4 \mathrm{~mm}$ on gold standard stress views. However, $0 / 9$ at most recent follow up have had MCS $>4 \mathrm{~mm}$ and therefore none have shown signs indicative of a need for surgery.

Hypothesis: The gold standard of stress views for isolated fibular fractures may be misrepresenting the common biomechanical stressors carried through the ankle as part of activities of daily living. Therefore, we believe it is possible that many isolated fibular fractures are being unnecessarily treated with operative intervention.

https://doi.org/10.32873/unmc.dc.gmerj.1.1.039

\section{Effect of Crystalloid Infusion Rate in a Noncompressible Hemorrhage Model} Ujwal Yanala, Jason Johanning, Iraklis Pipinos, Gustavo Larsen, William Velander, Mark Carlson

\section{Mentor: Mark Carlson \\ Program: General Surgery}

Purpose: To determine the effect of fluid administration rate on survival, vital signs, blood loss, and laboratory parameters in a porcine model of noncompressible hemorrhage.

Methods: Twenty domestic swine (barrow, age 3 months, 32-36 kg) underwent hemitransection of left lateral liver lobe without treatment. Incision was temporarily closed immediately after injury. At 60's after injury, lactated ringers solution was begun at either 150 or $20 \mathrm{~mL} / \mathrm{min}$ IV (rapid and slow group, respectively, $\mathrm{N}=10$ each); maximum volume was capped at $100 \mathrm{~mL} / \mathrm{kg}$. Both groups were monitored for 60 or $180 \mathrm{~min}$.

Results: Pre-injury physiological parameters did not differ between the groups. Survival after one hour in both the groups was $60 \%$; no further death occurred in the slow group with observation out to $180 \mathrm{~min}$. Necropsy demonstrated that an equivalent number of portal vein and hepatic vein branches had been transected in each group. There were no significant differences between groups for heart rate, temperature, total volume of LR infused, or liver weight.

Conclusion: Although the two groups were not directly comparable (no formal randomization; longer observation time in the slow group), this study demonstrated that in a porcine model of noncompressible truncal hemorrhage, intravenous crystalloid resuscitation with a relatively slow infusion rate $(20 \mathrm{~mL} / \mathrm{min})$ produced less blood loss and an improved laboratory profile (hemoglobin and protime). This study supports the U.S. military's recent adoption of a "hypotensive resuscitation" protocol for warfighters injured in the field, which dictates that fluid resuscitation of an injured warfighter with hemorrhagic shock should be restricted until the subject arrives at a forward surgical unit.

https://doi.org/10.32873/unmc.dc.gmerj.1.1.040

\section{Renal Salvage after Stent Graft Placement after Acute Renal Artery Occlusion with Prolonged Time of Ischemia}

Yue Gao, Dimitrios Miserlis, Matthew Longo, Nitin Garg

Mentor: Nitin Garg

Program: General Surgery

Purpose: To describe a patient with acute renal artery occlusion (RAO) who underwent successful revascularization procedure, after experiencing prolonged ischemic period, which resulted in successful retrieval of renal function.
Case Report: A middle-aged patient with past history left renal artery stenosis and stent graft placement presented with symptoms of chest pain, shortness of breath, and flank pain. The patient was admitted to Intensive Care Unit with diagnosis of multi-organ failure and subsequent anuria that led to initiation of hemodialysis. Computed tomography angiography (CTA) demonstrated chronic aortic occlusion along with bilateral proximal renal artery occlusion with reconstitution of the mid to distal renal arteries via collateralization. Patient underwent angioplasty with bilateral renal artery stent-graft placement and successful revascularization of proximal renal arteries. Post-operatively, renal function and urine output improved, and the patient was able 